\title{
DESIGN OF AN ELECTRO-OPTICAL SAMPLING EXPERIMENT AT THE AWA FACILITY
}

\author{
J. Ruan, H. Edwards, Cheng-Yang Tan, R. Thurman-Keup, V. Scarpine, FNAL, Batavia, USA \\ 60510
}

Y. Li, John Power, ANL, Argonne, IL 60439, U.S.A.

Tim Maxwell, Northern Illinois University, De Kalb, IL 60115, U.S.A.

\begin{abstract}
The free space electro-optical (EO) sampling technique is a powerful tool for analyzing the longitudinal charge density of an ultrashort e-beam. In this paper, we present (i) experimental results for a laser-based mock-up of the EO experiment [1] and (ii) a design for a beam-based, single-shot, EO sampling experiment using the e-beam from the Argonne Wakefield Accelerator (AWA) RF photoinjector. For the mock-up, a tabletop terahertz experiment is conducted in the AWA laser room. The mock-up uses an IR beam incident on $<110>$ ZnTe crystal to produce a $\mathrm{THz}$ pulse via optical rectification. Detection is based on the cross correlation between the $\mathrm{THz}$ field and the probe IR laser field in a second $<110>$ ZnTe crystal.
\end{abstract}

\section{INTRODUCTION}

With the advance of electron acceleration techniques, the bunch length has become shorter over the decades. Various techniques has been used to measure such ultra short bunches. One of the common techniques is the analysis of the incoherent transition radiation using a streak camera. The resolution of this technique is limited by the resolution of the streak camera. A transverse deflecting structure (LOLA) is another powerful tool to do this measurement and the resolution can be as short as 100 fs. [2] However both techniques are invasive methods which make them less useful for real time monitoring.

EO sampling is proving to be a very powerful tool for longitudinal beam diagnostics. [1,3-7] In particular, it is a non-destructive method compared to the other techniques, which makes it very attractive for real time monitoring. EO techniques are based on the principle of the pockel cell effect. When an electric field is applied to a birefringent crystal, the refractive index ellipsoid will change, introducing an optical phase shift. To detect this phase shift, an optical beam polarized at $45^{\circ}$ to the transverse axes of the EO crystal is propagated through the EO crystal during the presence of the electric field. The phase retardation is converted to an intensity modulation after a crossed polarizer. Recently several single shot schemes have been developed to detect an ultrashort e-bunch down to 150 fs. Comparative studies show a good agreement between EO techniques and LOLA measurements.[8]
In this paper we will report on the effort to build an EO sampling experiment at the Argonne Wakefield Accelerator (AWA) lab. We will describe the mock-up experiment done using a femtosecond laser source first. Then we will discuss the ongoing EO experiment with a picosecond laser source. Finally we will discuss the difference between these two measurements.

\section{EXPERIMENT}

\section{Experiment with femtosecond laser}

The schematic of the mocked-up femtosecond laser experiment is shown in figure 1 . The $70 \mathrm{fs}, 0.78 \mathrm{~mJ}$ laser output of a Ti:sapphire regen amplifier system with the repetition rates of $1 \mathrm{kHz}$ is split into a probe beam and pump beam. The pump beam, which carries $90 \%$ of the energy from the seed pulse, drives a 3mm thick ZnTe crystal with (001) cut producing a terahertz (THz) generator. The laser spot size is $8 \mathrm{~mm}$ on the crystal surface. A parabolic mirror is used to focus the terahertz onto a 1mm thick ZnTe (001) sampling crystal. A singleshot detection technique based on the cross correlation between the terahertz field and the probe laser pulse is adopted [9]. The sampling crystal is sandwiched between a pair of crossed polarizers with the probe beam imaged onto a charge-coupled device (CCD) camera. The setup has an extinction ratio better than $10^{6}$ before introducing the sampling crystal and drops to 3500 after the crystal is inserted due to the residual birefringence in the sampling ZnTe crystal.

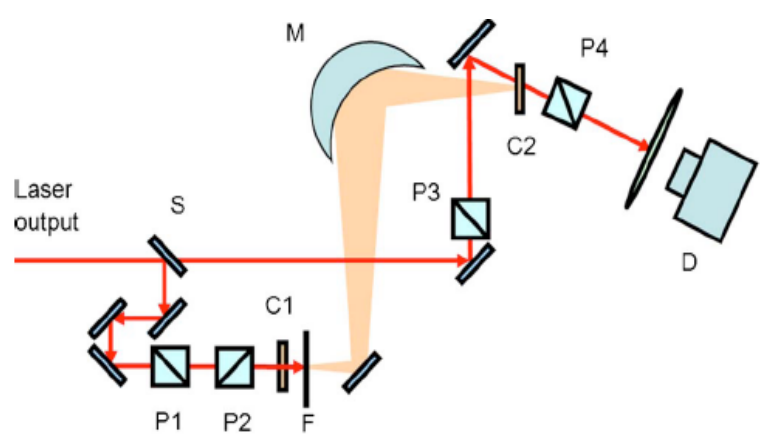

Figure1: Schematic of the experiment. P, C, F, M, D, S stands for polarizer, crystal, teflon filter, parabolic mirror, detector (CCD camera) and beam splitter respectively. More details about this experiment can be found in Ref. 1. 

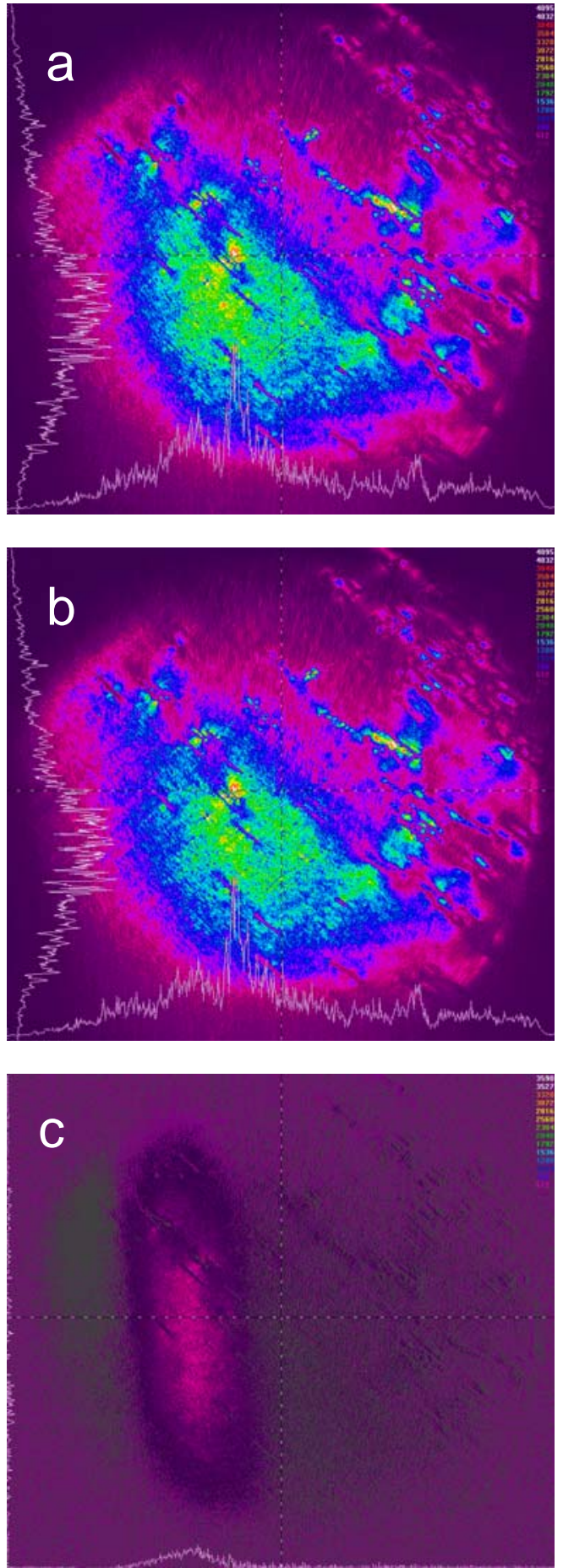

Figure 2 Experimental EO data using the fs laser generated terahertz. (a) is the background image with only probe pulse. (b) is the raw image with the presence of both pump and probe beam. (c) is the background subtracted version of (b).

Another pair of polarizers are inserted in front of the emitting crystal in order to adjust the laser intensity while maintaining the polarization on the emitting crystal.

Figure 2 shows a series of pictures taken at our experiment condition. The pulse energy used to generate the $\mathrm{THz}$ is $0.4 \mathrm{~mJ}$ and the probe signal is about $0.1 \%$ of the pump signal. The top picture is the background without the $\mathrm{THz}$ generation. This non-dark background is caused by the residual birefringence of the sampling crystal as stated before. The middle picture is the raw picture taken when both pump and probe are present and overlapped in the time domain. If we do a simple subtraction of the background from this picture we will get picture $\mathrm{C}$, which clearly shows the spatial trace caused by the $\mathrm{THz}$ wave. More details of this experiment can be found in Ref 1.

\section{Experiment with picosecond laser}

So far we have discussed the mock up experiment using a femtosecond laser pulse. In order to mimic the beam condition in AWA, we used a picosecond laser source to do another mock up experiment. The setup is shown in figure 3 . The femtosecond seed laser from a Ti-sapphire oscillator ( $\lambda=744 \mathrm{~nm}, \Delta \lambda=5 \mathrm{~nm}$ ) was split into 2 equal intensity pulses. The first pulse was used as our probe beam and traveled along the dashed line shown. The second pulse was sent into a stretcher, where its bandwidth was reduced to $1 \mathrm{~nm}$ and into the REGEN where it was amplified to the $\mathrm{mJ}$ level. The repetition rate for the regen-amplifier is $10 \mathrm{~Hz}$. The output from the regen amplifier was used as our picosecond pump to generate the $\mathrm{THz}$ wave. A pockel cell unit was used to lower the repetition rate of the probe pulse from 81.25 $\mathrm{MHz}$ to $10 \mathrm{~Hz}$ and to select the appropriate pulse to overlap in the time domain with the picosecond pump pulse.

A fast diode, placed at the location of the sampling crystal, and a $15 \mathrm{GHz}$ Tektronix scope were used to coarsely align the pump and probe pulses to within 250 picoseconds. We then placed the crystal back into the setup and observed the CCD camera for the signal while a computer controlled stage swept a 300 ps range. So far we have not observed any noticeable change of the image as was seen in the femtosecond laser experiment. One possible problem is the limited resolution of the coarse temporal alignment. A laser correlation measurement using a BBO crystal is planned to obtain a finer temporal alignment.

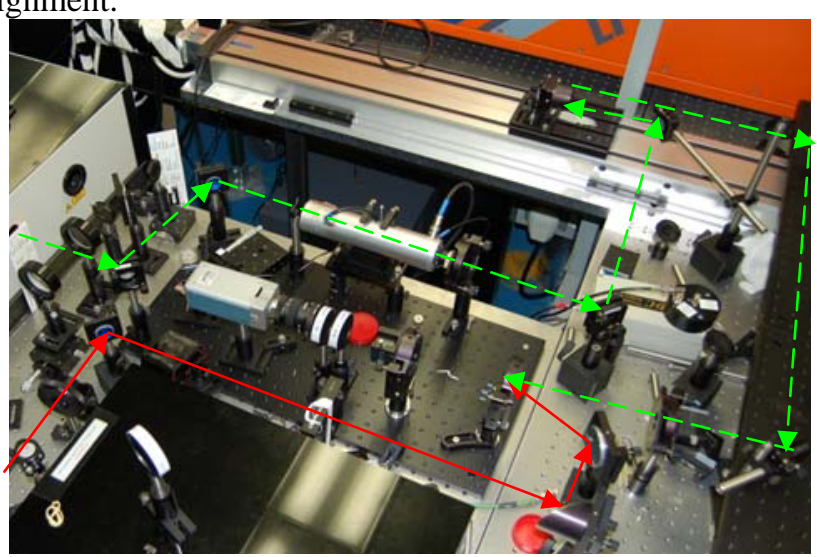

Figure 3: Experiment setup of the picosecond laser induced THz EO sampling. The solid red line is the THz generation and propogation direction. The dashed green line is the direction that probe beam travels. 


\section{DISCUSSIONS}

The main difference between our femtosecond and picosecond EO experiments are the $\mathrm{THz}$ generation parts. The detection scheme is almost identical except for the angle between the probe light and $\mathrm{THz}$ propagation directions. This difference arises from the need to have different time windows for each experiment.

In most $\mathrm{THz}$ generation studies, a femtosecond laser is used as the pump beam. There are two major reasons for this. First, just as with frequency doubling, $\mathrm{THz}$ generation through optical rectification is also a nonlinear process, which means that the larger peak intensities in shorter pulses will lead to much larger $\mathrm{THz}$ intensities. Secondly, since femtosecond lasers have a larger bandwidth, the generated pulse has bandwidth that extends from 0 to several $\mathrm{THz}$. On the other hand, the 10 picosecond pulse used at the AWA may only have a bandwidth extending out to several hundred GHz.

In our experiment the intensity issue can be compensated by increasing the pump pulse energy and focusing the beam to a smaller spot at the same time. However, the bandwidth issue will still limit the upper bandwidth of the signal, which means that the THz signal that we will be able to detect is much smaller in comparison with the femtosecond laser induced $\mathrm{THz}$ signal.

During the course of our experiment we have tried unsuccessfully to use both a pyroelectric and golay cell to directly detect the picosecond laser generated THz. When we compare the response curves of both detectors to the expected spectrum of our picosecond laser induced (few 100 's GHz), we find that the response of both detectors rolls off at the point where the induced spectrum turns on. This explains our failure to detect the generated signal in this case.

We expect a much better sensitivity with EO sampling than with the conventional detectors, even at lower repetition rate there are several issues which might need special attention. For example the background issue will be much more pronounced in the ps laser experiment than fs laser experiment.

\section{ACKOWLEDGEMENT}

This work is supported by Fermi Research Alliance LLC. under DE-AC02-07CH11359 with the U.S. DOE. T. M. is supported by DOE under Contract No. DE-FG02-06ER41435 with NIU.

\section{REFERENCES}

[1] Yuelin Li, "Electrical-optical sampling at near-zero optical bias” Appl. Phys. Letts. 88 (2006), 251108
[2] M. Röhrs, C. Gerth and H. Schlarb, "Invesitgations of the longitudinal electron bunch structure at the FLASH linac with a transverse deflecting RFstructure”, Proceedings of FEL 2006, Berlin 06, TUBAU06

[3] X. Yan, A. M. Macleod, W. A. Gillespie, G. M. H. Knipples, D. Oepts, A. F. G. van der Meer, and W. Seidel, "Subpicosecond Electro-optic Measurement of Relativistic Electron Pulses”, Phys. Rev. Lett. 85 (2000) 3404.

[4] I. Wilke, A. M. Macleod, W. A. Gillespie, G. Berden, G. M. H. Knippels and A. F. G. van der Meer, "Single-Shot Electron-Beam Bunch Length Measurements”, Phys. Rev. Lett. 88, (2002) 124801

[5] G. Berden, S. P. Jamison, A. M. Macleod, W. A. Gillespie, B. Redlich, and A. F. G. van der Meer, "Electro-Optic Technique with Improved Time Resolution for Real-Time, Nondestructive, SingleShot Measurements of Femtosecond Electron Bunch Profiles”, Phys. Rev. Lett. 93, (2004) 114802

[6] A. L. Cavalieri, D. M. Fritz, S. H. Lee, P. H. Bucksbaum, D. A. Reis, J. Rudati, D. M. Mills, P. H. Fuoss, G. B. Stephenson, C. C. Kao, D. P. Siddons, D. P. Lowney, A. G. MacPhee, D. Weinstein, R.W. Falcone, R. Pahl, J. Als-Nielsen, C. Blome, S. Du“sterer, R. Ischebeck, H. Schlarb, H. SchulteSchrepping, Th. Tschentscher, J. Schneider, O. Hignette, F. Sette, K. Sokolowski-Tinten, H. N. Chapman, R.W. Lee, T. N. Hansen, O. Synnergren, J. Larsson, S. Techert, J. Sheppard, J. S. Wark, M. Bergh, C. Caleman, G. Huldt, D. van der Spoel, N. Timneanu, J. Hajdu, R. A. Akre, E. Bong, P. Emma, P. Krejcik, J. Arthur, S. Brennan, K. J. Gaffney, A. M. Lindenberg, K. Luening, and J. B. Hastings, “Clocking Femtosecond X rays”, Phys. Rev. Lett. 94 (2005) 114801.

[7] T. Tsang, V. Castillo, R Larsen, D. M. Lazarus, D. Nikas, C. Ozben, Y. K. Semertzidis, T.SrinivasanRao and L. Kowalski, "Electro-optical measurements of picosecond bunch length of a $45 \mathrm{MeV}$ electron beam”, J. Appl. Phys. 89, (2001) 4921

[8] A. Azima, S. Düsterer, M. Hüning, E.-A. Knabbe, M. Roehrs, V. Rybnikov, H. Schlarb, B. Schmidt, B. Steffen, P.Schümser, A. Winter, M. Ross, "Comparative study of bunch length and arrival time measurements at FLASH”, EPAC’06 Edingburgh 06, 1049

[9] S. Jamison, J. Shen, a. M. Macleod, W.A. Gillespie, and D. A. Jaroszynski, "High-temporal-resolution, single-shot characterization of terahertz pulses”, Opt. Lett. $25 \quad$ (2003), 1710 